\title{
Potential for nosocomial transmission of multidrug-resistant (MDR) tuberculosis in a South African tertiary hospital
}

\author{
Colleen M Bamford, Jantjie J Taljaard
}

Background. Tuberculosis (TB) is a major health problem in the Western Cape, with an incidence exceeding 900 per 100000 people. Nosocomial transmission of $\mathrm{TB}$, and particularly drug-resistant $\mathrm{TB}$, is a potential risk that may be undetected. Rapid diagnosis and rapid institution of effective anti-TB treatment, combined with appropriate infection control measures, are essential to prevent nosocomial transmission of TB. To estimate the potential for nosocomial transmission, we aimed to determine the in-hospital delays in diagnosis and treatment of patients with multidrug-resistant (MDR)-TB at a tertiary care hospital.

Methods. A descriptive study, based on retrospective review of patient records and laboratory data, including all adult patients ( $>13$ years) where TB culture and susceptibility testing confirmed MDR-TB on specimens submitted to Tygerberg Hospital's National Health Laboratory Service (NHLS) laboratory in 2007.
Results. Thirty-one patients with MDR-TB were identified. The median laboratory turnaround time (TAT) from collection of specimen to confirmation of MDR-TB was 40 days, while the median time from the time of first presentation at Tygerberg Hospital to institution of MDR treatment was 44 days. Twenty patients were considered infectious during their hospital stay, generating 345 inpatient infectious days.

Conclusions. The study suggests that there is an ongoing substantial risk for nosocomial transmission of MDR-TB at Tygerberg Hospital. We propose improvements, including the use of rapid drug susceptibility testing. The consistent application of infection control measures to prevent nosocomial spread of TB, including MDR-TB, remains vital.

S Afr Med J 2010; 100: 438-441.
Tuberculosis (TB) is a major health problem in the Western Cape, with a rising incidence which now exceeds 900 per 100000 population. ${ }^{1}$ In this setting, nosocomial transmission of TB is a potential risk, particularly to HIV-infected or other immunocompromised patients. Nosocomial outbreaks of TB are usually noted initially because of increasing rates of TB among previously hospitalised patients, and subsequently confirmed by molecular typing of Mycobacterium tuberculosis isolates. However, in high-incidence areas, detection is hampered by the high background level of TB within communities, delays in development of symptoms in immunocompetent persons, and the existence of multiple TB service providers. In South Africa, nosocomial outbreaks of TB have been documented among HIV-infected patients at Sizwe Tropical Disease Hospital, ${ }^{2}$ neonates in a kangaroo mother care unit, ${ }^{3}$ and in a rural hospital at Tugela Ferry. ${ }^{4}$ This latter outbreak involved the spread of extensively drug-resistant (XDR) TB (defined as resistance to isoniazid, rifampicin, any fluoroquinolone and at least 1 of 3 injectable second-line drugs) ${ }^{5}$ among a large number of HIV-infected patients with rapidly fatal outcomes. Detection of these outbreaks was

National Health Laboratory Service, Tygerberg Hospital, and Department of Medical Microbiology and Immunology, Stellenbosch University, Tygerberg, W Cape

Colleen M Bamford, MB ChB, MMed (Med Micro), FCPath (SA), MPhil, Dipl Child Health (current affiliation: NHLS Groote Schuur Hospital and Department of Medical Microbiology, University of Cape Town)

Division of Infectious Diseases, Department of Medicine, Tygerberg Hospital, and Centre for Infectious Diseases, Stellenbosch University

Jantjie J Taljaard, MB ChB, MMed(Intern), DTM\&H facilitated by the vulnerable status of the patients, which resulted in rapid progression to disease, drug-resistant status of infecting strains, and the relative isolation of the hospitals in terms of geography or timing of exposure. It is likely that cases of nosocomial TB remain undetected in other situations.

Multidrug-resistant (MDR)-TB (defined as TB resistant to both isoniazid and rifampicin) is more difficult to treat than drug-sensitive $\mathrm{TB}$, requires longer courses of more toxic drugs, and has poorer outcomes. Therefore, there is particular concern regarding the transmission of MDR-TB within health care facilities in South Africa. Rapid diagnosis and rapid institution of effective anti-TB treatment, combined with appropriate infection control measures, are key factors in the prevention of nosocomial transmission of TB.

We aimed to determine the in-hospital delays in diagnosis and treatment of patients with MDR-TB at Tygerberg Hospital (an academic tertiary care hospital) over the period of 1 year, to estimate the potential for nosocomial transmission.

\section{Methods}

This was a descriptive study based on retrospective review of patient records and laboratory data, and included all adult patients (>13 years) in whom TB culture and susceptibility testing confirmed MDR-TB on specimens submitted to Tygerberg Hospital National Health Laboratory Service (NHLS) between 1 January and 31 December 2007. The decision to request TB culture and drug susceptibility testing (DST) was made by the attending clinician. Culture and susceptibility testing was performed in an automated liquid culture system (MGIT, Becton Dickinson, Sparks, Maryland) and confirmation of $M$. tuberculosis was by a rapid molecular method. ${ }^{6}$ All tests were carried out in an accredited laboratory using appropriate safety precautions. 
Patient records were retrieved and data extracted using a standardised form. The dates of any outpatient visits and inpatient admissions were recorded; results of TB investigations, including DST results, and the dates on which such results were available, were obtained from the laboratory information system databases of the NHLS laboratories at Tygerberg and Green Point. Use of the Green Point database provided access to the results of any specimens sent from other hospitals or clinics in the 3 months preceding presentation at Tygerberg Hospital.

Clinical failure was defined as persistence of symptoms after 2 months of first-line TB therapy. Microbiological failure was defined as failure of smear conversion (in those initially smear-positive) after 2 months of first-line TB therapy. For the calculation of in-hospital infectivity, patients were assumed to be infectious from the date of admission until the date when they started MDR treatment.

The study was approved by the Committee for Human Research of the Faculty of Health Sciences, University of Stellenbosch (N08/03/064). The Department of Infectious Diseases at Tygerberg Hospital undertook to contact all patients discharged home who lacked evidence of commencement of MDR treatment.

\section{Results}

From the 355 adults with culture-confirmed tuberculosis in whom DST was performed, 31 patients with MDR-TB were identified and folders retrieved; there were 21 males and 10 females, and their median age was 32 years (range 18 - 53 years). The majority of patients $(20 / 31)$ were referred to Tygerberg Hospital (TBH) and originated from a variety of sources (Table I). Four patients were already attending the hospital's Infectious Diseases Clinic, referred initially in most cases to access antiretroviral therapy (ART). One staff member presented to the occupational health clinic.

The majority (26/31) of patients were admitted. Only 5 were seen exclusively on an outpatient basis, including the staff member seen at the occupational health clinic, an 18-year-old known MDR patient receiving chronic care from the paediatric service, a known MDR patient referred for surgical assessment, a known MDR defaulter who self-referred, and 1 patient diagnosed with TB in the emergency ward, where the duration of her stay was unclear.

Five patients (included in the 9 referred from other hospitals) were referred from G F Jooste Hospital to the Khayelitsha

Table I. Origin of adult multidrug-resistant tuberculosis (MDR-TB) patients at TBH in 2007

\begin{tabular}{lrr}
\hline Origin & $N$ & $\%$ \\
\hline Day hospital/community health centre/TB clinic & 7 & 23 \\
Other hospital (not TB hospital) & 9 & 29 \\
General practitioner & 4 & 13 \\
TBH Infectious Diseases Clinic & 4 & 13 \\
TBH Occupational Health Clinic & 1 & 3 \\
TBH Paediatric TB Clinic & 1 & 3 \\
Self-referred & 2 & 6 \\
Unknown & 3 & 10 \\
$\quad$ Total & 31 & 100 \\
\hline
\end{tabular}

District Hospital, located within the TBH complex. Four of these 5 patients were either known or suspected MDR patients who were awaiting placement in the MDR section of Brooklyn Chest Hospital (BCH).

\section{Presence of known risk factors for MDR-TB}

Six patients were known to have MDR-TB at the time of referral/presentation and were referred with complications of TB (2), for assessment for surgery (1), to await placement at $\mathrm{BCH}$ (1) or self-referred (1). One additional patient had been treated by the paediatric service for MDR-TB for a number of years.

MDR-TB was listed as part of the initial differential diagnosis in $8(32 \%)$ of the remaining 25 patients. All 8 had evidence of clinical or microbiological failure at the time of presentation. An additional 4 patients had evidence of clinical or microbiological failure on presentation, but MDR-TB was not listed in the differential diagnosis. It was not possible to determine retrospectively any specific reasons for the requesting of DSTs in the remaining 13 patients.

Eighteen (58\%) of the 31 patients gave a history of previous $\mathrm{TB}$; in $4(13 \%)$ this was their first episode of TB, and in 9 $(29 \%)$ no details were recorded. Microbiological details of the previous episode of TB were not available in most cases (susceptibility results available only in 5 known MDR patients, while 2 other patients had previous culture-proven TB without susceptibility testing, and 1 patient had been shown to be smear-negative on completion of previous therapy).

In 2 cases, the patients reported previous contact with a person with known MDR-TB. One of these cases was one of the 6 patients known to have MDR-TB at presentation to $\mathrm{TBH}$. It was not possible to assess whether patients had previously defaulted from TB treatment, as this information was seldom recorded.

\section{HIV co-infection}

HIV status was unknown in 4 patients. Of the 27 patients whose HIV status was known, 16 (59\%) were HIV negative and $11(41 \%)$ were HIV positive. At the time of diagnosis of MDRTB, 7 HIV-positive patients were receiving or had previously received ART.

\section{Site of infection}

Twenty-six patients $(84 \%)$ had pulmonary TB (PTB), while 5 had extra-pulmonary $\mathrm{TB}$, including 3 with pleural disease, 1 with arthritis and 1 with lymphadenitis. Of the patients with pleural TB, 1 was considered infectious owing to a broncho-pulmonary fistula with acid-fast bacilli (AFB) seen on microscopy of the pleural fluid. Twenty $(77 \%)$ of the 26 patients with PTB were smear-positive.

\section{Laboratory turnaround time}

The median laboratory turnaround time (TAT) from collection of specimen to confirmation of MDR-TB was 40 days (minimum 25 days, maximum 101 days, inter-quartile range (IQR) 29 - 62 days). The median TAT for second-line susceptibility testing was (an additional) 42 days, with a minimum of 30 days, maximum of 58 days and IQR of $35-46$ 
days. Only 7/31 (23\%) patients had MDR-TB confirmed by laboratory testing within 30 days.

\section{Second-line susceptibility testing and XDR-TB}

Isolates from 20 patients had second-line susceptibility testing with varying numbers of isolates being tested against each drug (Table II). Resistance rates ranged from $5 \%$ for ethionamide to $46 \%$ for ethambutol. Resistance rates to amikacin and ofloxacin were $19 \%$ and $25 \%$ respectively.

Results from 3 patients demonstrated resistance to ofloxacin and amikacin, i.e. XDR-TB; 2 had known MDR-TB, and 1 had a history of previous TB and subsequently died of presumed HIV encephalopathy (see following section). Of the remaining 17 patients, XDR was excluded in 10, while 2 showed resistance to an additional agent without fulfilling criteria for $\mathrm{XDR}$, and testing was incomplete in 5 .

\section{Mortality}

Five patients died in TBH within 2 weeks of admission, all before diagnosis of MDR-TB (crude mortality rate 16\%); 2 were HIV positive with advanced disease, and 3 were HIV negative. One HIV-negative patient who had been diagnosed 5 months previously at TBH with TB arthritis was re-admitted shortly before death with PTB. Drug susceptibility testing of the respiratory specimen confirmed MDR-TB (and excluded XDR-TB). As DST was not performed on the original tissue specimen, it was not possible to confirm whether he had MDRTB initially.

Another HIV-negative patient was already under investigation at a peripheral clinic for drug-resistant TB. Although MDR results were available 49 days prior to his death, no MDR treatment had been started. His isolate was also resistant to ethambutol and ofloxacin, but the diagnosis of XDR could not be confirmed or excluded since no further testing was done.

One HIV-positive patient who died of presumed HIV encephalopathy had XDR-TB. There was no evidence of central nervous system (CNS) tuberculosis on CSF examination and culture or on CT scan prior to death.

Of the remaining 2 patients who died, XDR-TB was excluded in one and second-line testing was not carried out for the other.

\section{Time to institution of MDR treatment at TBH}

Ten patients were not eligible to start MDR treatment as they were already on MDR treatment at presentation (5) or died before the diagnosis of MDR-TB (5). Of the remaining 21

\section{Table II. Second-line susceptibility test results of} submitted isolates

\begin{tabular}{llc}
\hline & $\begin{array}{l}\text { No. resistant/ } \\
\text { No. tested }\end{array}$ & $\%$ \\
\hline Ethambutol & $12 / 26^{*}$ & 46 \\
Amikacin & $3 / 16$ & 19 \\
Ofloxacin & $5 / 20$ & 25 \\
Ethionamide & $1 / 19$ & 5 \\
*An additional 6 isolates were tested for ethambutol susceptibility, but not for second- \\
line agents.
\end{tabular}

patients, 10 were started on MDR treatment at TBH and 11 were discharged on standard TB therapy (6) or transferred to $\mathrm{BCH}$ on standard TB therapy (5). Among the 10 patients with known date of starting MDR treatment, the median time to institution of treatment from the time of first presentation at TBH was 44 days (range 5 - 187 days).

\section{Timing of initiation of MDR treatment in relation to availability of DST results}

The time period between the availability of DST results confirming MDR-TB and the initiation of MDR treatment was calculated. For this calculation, results from specimens submitted from other institutions in the 3 months preceding presentation at Tygerberg Hospital were included (Table III).

Four patients were started on MDR treatment empirically based on clinical grounds, prior to the availability of DST results, supported by histological results (1 case) and by rapid molecular testing (1 case) accessed through research studies, as rapid molecular testing was not available as a diagnostic test in NHLS laboratories at the time.

Six patients were started on MDR treatment after DST results became available. In 3 patients, there was a delay of more than 30 days between availability of DST results and initiation of MDR-TB treatment.

\section{Period of in-hospital infectivity (TBH only)}

Patients were assumed to be infectious from the date of admission until they started MDR treatment. Twenty patients were considered infectious, as those patients already on MDR treatment (4), patients with extra-pulmonary TB (3) and patients treated as outpatients (4) were excluded. These 20 patients generated 345 inpatient infectious days averaging 17 days per patient. It proved impossible to determine retrospectively whether appropriate infection control measures including placement in an individual room or the use of personal protective equipment such as N95 respirators (masks) were applied to suspected or confirmed MDR-TB patients.

\section{Laboratory cross-contamination}

No evidence of laboratory cross-contamination was found. There was no clustering of MDR cases within the laboratory. In all patients, $\mathrm{TB}$ was confirmed by correlation with clinical findings, microscopy or histology results, or by isolation of mycobacteria from other specimens. However, susceptibility testing was not usually carried out on more than one specimen per patient, and no molecular fingerprinting tests were carried out.

\begin{tabular}{|c|c|c|}
\hline Number of days & $\begin{array}{l}\text { No. of } \\
\text { patients }\end{array}$ & $\begin{array}{l}\text { Exact } \\
\text { timing } \\
\text { (days) }\end{array}$ \\
\hline$>30$ days before results available & 4 & $33,33,34,95$ \\
\hline 0 - 7 days after results available & 1 & 4 \\
\hline 7 - 30 days after results available & 2 & 13,25 \\
\hline$>30$ days after results available & 3 & $86,98,132$ \\
\hline
\end{tabular}




\section{Discussion}

This small group of 31 patients with culture-confirmed MDRTB at a single tertiary care institution in 2007 generated 345 inpatient infectious days, corresponding to one infectious MDR patient in the hospital almost every day of the year. There were also considerable in-hospital delays in the microbiological diagnosis of MDR-TB (median 40 days) and the initiation of MDR treatment (median 44 days from first presentation at $\mathrm{TBH}$ ). However, the accuracy of this latter finding is limited by lack of data, as the date of commencement of MDR treatment was available for only 10 of 21 eligible patients, reflecting that many patients were discharged or transferred prior to the availability of laboratory results.

Only $23 \%$ of DST results were available within 30 days, ${ }^{7}$ a target suggested by the Centers for Disease Control and Prevention (CDC) prior to recent concerns about the increasing prevalence of drug resistance. While these TATs may not be an accurate reflection of laboratory performance, as DST is sometimes only requested by clinicians some time after receipt of positive culture results, it suggests that current phenotypic methods of DST, including automated methods, are inadequate in situations of increasing drug resistance. Rapid DST should therefore be more widely available to expedite management of suspected MDR-TB patients, and possibly for all hospitalised PTB patients at the time of diagnosis. At the time of the study, second- and third-line DST was not routinely performed for all MDR patients, making interpretation of additional resistance patterns impossible. Provincial policy has subsequently been amended to allow for automatic second-line testing for all MDR isolates.

While a shorter laboratory TAT can accelerate diagnosis and initiation of treatment, failure of health care workers to respond to laboratory results is another source of delay. The long delay of more than 1 month in 3 of 10 patients with known date of starting treatment is of concern. Part of this delay could be because clinicians were unaware of or unable to access the results of specimens submitted from other institutions. However, lack of patient co-operation might have contributed to delays, e.g. if a patient failed to return for follow-up at a peripheral hospital or clinic.

Given the slow laboratory TAT, it is not surprising that that the period of potential in-hospital infectivity was high. The figure of 345 inpatient infectious days is likely to be an underestimate of infectivity, as patients were assumed to become non-infectious immediately on commencement of MDR treatment. While the implementation of appropriate infection control measures could mitigate the possibility of nosocomial transmission, it was not possible to assess such measures retrospectively. However, infection control measures are inconsistently applied in this setting ${ }^{8}$ and there is consequently a considerable risk for nosocomial transmission of MDR-TB.

The study included only those MDR-TB cases confirmed on culture at TBH, excluding those diagnosed elsewhere, as well as those treated empirically for MDR-TB. However, there was no alternative record of MDR-TB patients at TBH (personal communication, Infection Prevention and Control Unit), highlighting a shortcoming in the current system of MDR management. In addition, since culture and DST are performed only for a minority of TB cases, it is likely that this study under-estimates the number of cases of MDR-TB.

The present study did not include patients with rifampicin mono-resistance, although this is recently of increasing concern in the Western Cape. It was not practical to compare laboratory-based resistance rates from previous years because the changing pattern of DST requesting, with an increase in the number of DST requests, is likely to introduce bias and complicate interpretation. Limiting the study to a single institution also made it difficult to analyse patients who frequently move between institutions within the wider health care system.

The study suggests that there is an ongoing substantial risk for nosocomial transmission of MDR-TB at one local tertiary institution. While the incidence of drug-resistant TB may be less at primary- or secondary-level hospitals, they are likely to face similar delays in microbiological diagnosis. Steps that could be taken to reduce delays in diagnosis include the use of rapid DST, and the provision of improved access for clinicians to national or provincial laboratory information systems to facilitate retrieval of results from other institutions. The consistent application of infection control measures to prevent nosocomial spread of TB (including MDR-TB) remains vital.

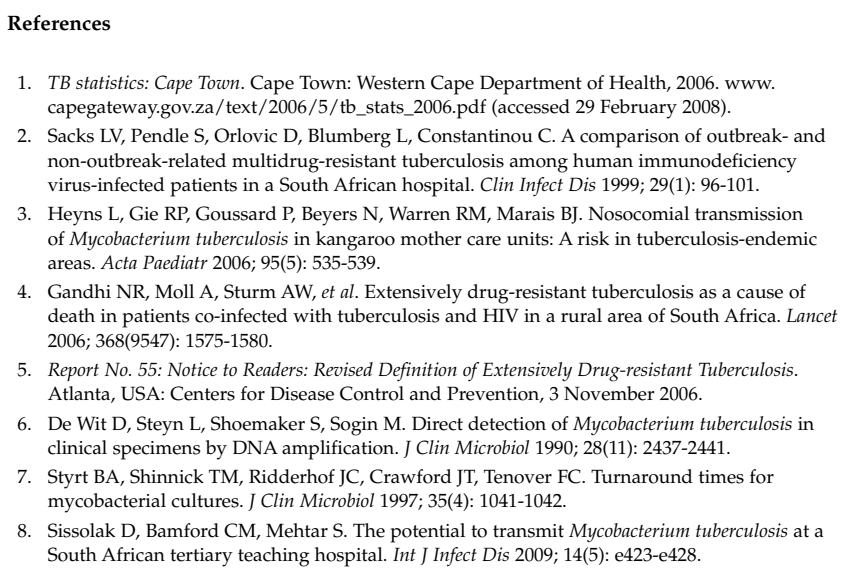
capegateway.gov.za/text/2006/5/tb_stats_2006.pdf (accessed 29 February 2008).

2. Sacks LV, Pendle S, Orlovic D, Blumberg L, Constantinou C. A comparison of outbreak- and non-outbreak-related multidrug-resistant tuberculosis among human immunodeficiency virus-infected patients in a South African hospital. Clin Infect Dis 1999; 29(1): 96-101.

3. Heyns L, Gie RP, Goussard P, Beyers N, Warren RM, Marais BJ. Nosocomial transmission of Mycobacterium tuberculosis in kangaroo mother care units: A risk in tuberculosis-endemic of Mycobacterium tuberculosis in kangaro
areas. Acta Paediatr 2006; 95(5): 535-539.

4. Gandhi NR, Moll A, Sturm AW, et al. Extensively drug-resistant tuberculosis as a cause of death in patients co-infected with tuberculosis and HIV in a rural area of South Africa. Lancet 2006; 368(9547): 1575-1580.

5. Report No. 55: Notice to Readers: Revised Definition of Extensively Drug-resistant Tuberculosis. Atlanta, USA: Centers for Disease Control and Prevention, 3 November 2006.

6. De Wit D, Steyn L, Shoemaker S, Sogin M. Direct detection of Mycobacterium tuberculosis in clinical specimens by DNA amplification. J Clin Microbiol 1990; 28(11): 2437-2441.

7. Styrt BA, Shinnick TM, Ridderhof JC, Crawford JT, Tenover FC. Turnaround times for mycobacterial cultures. J Clin Microbiol 1997; 35(4): 1041-1042.

8. Sissolak D, Bamford CM, Mehtar S. The potential to transmit Mycobacterium tuberculosis at a South African tertiary teaching hospital. Int J Infect Dis 2009; 14(5): e423-e428.

Accepted 15 December 2009. 